\title{
Search for magnetically quiet CHAMP polar passes and the characteristics of ionospheric currents during the dark season
}

\author{
P. Ritter and H. Lühr \\ GeoForschungsZentrum Potsdam, Telegrafenberg, 14473 Potsdam, Germany
}

Received: 6 July 2006 - Revised: 22 September 2006 - Accepted: 19 October 2006 - Published: 21 November 2006

\begin{abstract}
The magnetic activity at auroral latitudes is strongly dependent on season. During the dark season, when the solar zenith angle in the polar region is larger than $100^{\circ}$ at all local times, the ionospheric conductivity is much reduced, and generally low activity is encountered. These time intervals are of special interest for the main field modelling, because then the geomagnetic field readings, in particular the field magnitude, are only slightly affected by ionospheric currents. Based on CHAMP data, this study examines how these quiet periods are reflected in the different magnetic field components. The peak FAC density is used as a possible proxy for the deviation of the total field. As a second option, the transverse field component, which is aligned with the auroral oval, is investigated, because it presents a measure for the FAC total current. Correlation analyses with the scalar residuals are performed and both proxies are tested for their suitability of predicting the intensity of the auroral electrojet during the dark polar seasons. The indicators based on the local FAC strength or on the amplitude of the transverse component show a reasonable correlation with the electrojet intensity for these periods, but fail when limited to small amplitudes. The predictability improves considerably if the time sector is limited to dayside hours (08:00-16:00 MLT). As the activity at high latitudes is strongly controlled by the solar wind input, we also consider IMF quantities which may support very quiet conditions. Correlations of the magnetic field scalar residuals with the merging electric field are strongest if only passes in the dayside sector are considered. Best selection results for quiet passes are obtained by combining four conditions: dark season, small average merging electric field, $E_{m}<0.8 \mathrm{mV} / \mathrm{m}$, absence of peak values of $E_{m}>1.2 \mathrm{mV} / \mathrm{m}$ during a time interval of $40 \mathrm{~min}$ centred at the polar crossing, and limitation to the dayside sector (08:00-16:00 MLT). The set of quiet polar passes identified by these criteria may be used beneficially in crustal field modelling of the polar regions.
\end{abstract}

Correspondence to: P. Ritter

(pritter@gfz-potsdam.de)
Keywords. Ionosphere (Auroral ionosphere; Electric fields and currents; Ionosphere-magnetosphere interactions)

\section{Introduction}

The auroral regions in both hemispheres are characterised by a wide range of magnetic activity. Main driver is the interaction of the solar wind with the magnetosphere. Processes taking place at the magnetopause or in the magnetospheric tail are coupled by field-aligned currents (FACs) to the highlatitude ionosphere. Furthermore, the intensity of the FACs can be influenced by the conditions in the ionosphere. Wang et al. (2005) showed that the conductivity induced by solar irradiation has a strong influence on the current strength on the day side. Conversely, on the night side, these authors found little dependence of the FAC intensities on the solar zenith angle. In this time sector the effect of precipitating electrons seems to play a dominating role for maintaining the ionospheric conductivity.

In this article we want to focus on periods with very weak currents. Such intervals are of particular interest for modelling studies of the internal magnetic field since ionospheric currents have to be considered as a source of noise. Ideally, all components would be used at all latitudes to account also for the ionospheric currents in the modelling. As this cannot be achieved, at high-latitude regions only the field magnitude is used in main field studies (e.g. Langel and Estes, 1985; Olsen, 2002). This is done in order to avoid the influence of large magnetic deflections caused by field-aligned currents. The total field, on the other hand, is affected predominantly by horizontal ionospheric currents, such as the auroral electrojet. Since the electrodynamics at auroral latitudes is driven primarily by field-aligned currents, there is a close relation expected between the electrojet strength and the FAC intensity.

It has been shown that standard magnetic activity indices like $K_{p}$ or $D_{s t}$ are not very efficient for selecting periods of quiet times at auroral latitudes (Ritter et al., 2004a). Slightly

Published by Copernicus GmbH on behalf of the European Geosciences Union. 


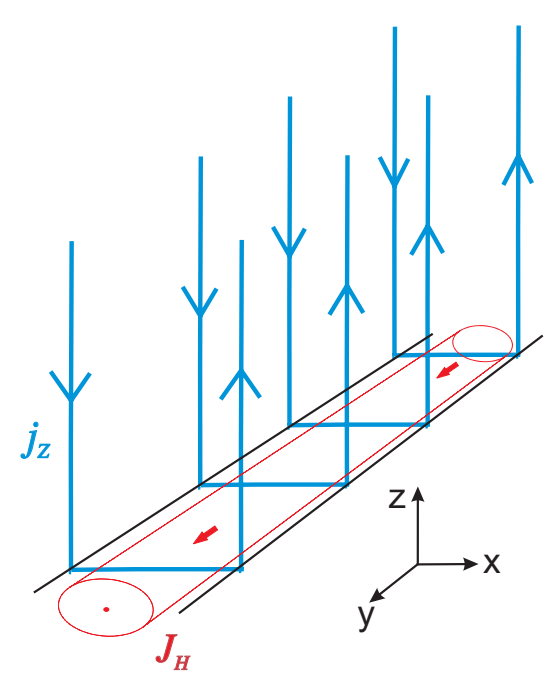

Fig. 1. Sketch of the 3-D geometry and coordinate system used here to describe the polar ionospheric current system.

better are the polar cap index, PC, or the auroral activity index, $A E$. These indices, however, reflect mainly the level of activity on the night side rather than on the day side. As has been demonstrated by Wang et al. (2005), quiet intervals on the day side are observed predominantly during the dark polar winter months. Another controlling factor is the coupling efficiency between the solar wind and the magnetosphere, which can reliably be described by the merging electric field, $E_{m}$, (Kan and Lee, 1979).

In this study we make use of five years of CHAMP magnetic field observations to find out the periods of low electrojet activity. Our aim is to identify the conditions prevailing during quiet intervals. The investigations presented here are regarded as an extension of the paper by Ritter et al. (2004a). In that study an attempt was made to predict the activity level during CHAMP polar passes based on solar wind conditions. The achieved success rate was ranging around 50\%. Here, we will test the efficiency of further local quantities like the FAC intensity or the deflection of the azimuthal field component as possible indicators for the strength of the auroral electrojet.

In the sections to follow we will outline the relations between the considered activity indicators and the electrojet strength, then examine their correlations and distributions with magnetic local time, and finally review their suitability to identify quiet polar passages.

\section{Characterisation of weak auroral currents}

The various types of auroral currents are coupled through the electric field and the ionospheric conductivity distribution. For a better assessment of their dependency some basic relations will be outlined here. We applied some simplifying assumptions. This allows us to use algebraic expressions for the quantities, and thus to reveal the functional relations between the currents.

At high latitudes, the ionospheric currents comprise Hall, Pedersen and field-aligned currents (FACs). All three contribute to the magnetic fields measured by satellites. As has been noted before, for main field modelling only the perturbations of the field magnitude are of concern. These deviations of the total field are produced by the toroidal current component, which is predominantly carried by Hall currents. It may thus be of interest to see how the Hall currents or the electrojet are related to the intensity of field-aligned currents. For our assessment we assume a pair of oppositely directed FAC sheets that are aligned with the auroral oval. The ionospheric conductivity is taken to be homogeneous, which is reasonable for quiet times. The FACs are then closed in the E-region by Pedersen currents, and Hall currents flow along the oval, in this case representing the electrojets. The anticipated current configuration is sketched in Fig. 1. For our calculations we use a coordinate system where the FACs are aligned with the $\mathrm{z}$ axis $\left(j_{z}\right)$, the electric field, $E$, is pointing in $\mathrm{x}$ direction, i.e. along the geomagnetic meridian, and the Hall current, $J_{H}$, is flowing in y direction. Under these conditions the relations between the currents can be expressed as

$$
\begin{aligned}
\nabla \cdot\left(\Sigma_{P} \mathbf{E}\right) & =-j_{z} \\
J_{H y} & =\Sigma_{H} E_{x}
\end{aligned}
$$

When combining Eqs. (1) and (2) we obtain

$\frac{\Sigma_{P}}{\Sigma_{H}} \frac{J_{H y}}{d x}=-j_{z}$

where $\Sigma_{H}$ and $\Sigma_{P}$ are the height-integrated Hall and Pedersen conductivities, respectively. Since the ratio between the conductances is rather constant, the meridional gradient of the Hall current reflects the distribution of the FAC density.

At auroral latitudes the magnetic inclination is almost $90^{\circ}$. Therefore, changes of the total field are caused primarily by the electrojet. The current density profile, $J(x)$, of the electrojet has often been approximated by a Lorentzian curve (e.g. Kertz, 1954; Maurer and Theile, 1978) which has the form

$J(x)=J_{0} \frac{a^{2}}{x^{2}+a^{2}}$

where $J_{0}$ is the peak current density and $2 a$ is the distribution width at half-peak value. For the spatial derivative of this distribution, which is proportional to the FAC density (cf. Eqn. 3), we obtain

$\frac{d J}{d x}=-2 J_{0} a^{2} \frac{x}{\left(x^{2}+a^{2}\right)^{2}}$

The magnetic deflection caused by a Lorentzian shaped current can be expressed analytically (e.g. Maurer and Theile, 
1978). For the vertical component, $b_{z}$, which reflects the modification of the field magnitude best, we obtain

$b_{z}=-\frac{\mu_{0} J_{0} a}{2} \frac{x}{x^{2}+(h+a)^{2}}$

where $h$ is the measurement height above the current sheet.

When comparing Eqs. (5) and (6) it can be seen that both have a similar dependence on the $\mathrm{x}$ component. This means a similar latitude profile is expected for the FAC density and the $b_{z}$ component. For the FACs the extremes are expected at $x_{m}= \pm a$ and for $b_{z}$ at $x_{m}= \pm(h+a)$.

$j_{z_{\max }}= \pm \frac{\Sigma_{P}}{\Sigma_{H}} \frac{J_{0}}{2 a}$

$b_{z_{\max }}= \pm \mu_{0} J_{0} \frac{a}{4(h+a)}$

Thus, for the given current geometry we can anticipate a linear relationship between the peak sizes in FACs and $b_{z}$.

In a similar way the electrojet can be characterised by the deflection of the $B_{y}$ component measured between the FAC sheets. When considering the geometry, as outlined in Fig. 1, the magnetic field generated by the FAC and Pedersen current circuit is limited to the y component and is termed $b_{y}$. The following relation holds under these conditions:

$j_{z}=-\frac{1}{\mu_{0}} \frac{d b_{y}}{d x}$

When considering Eq. (1) we may write

$$
\frac{1}{\mu_{0}} \frac{d b_{y}}{d x}=\nabla \cdot\left(\Sigma_{P} E\right)
$$

For the given geometry this term can be reduced to

$b_{y}=\mu_{0} \Sigma_{P} E_{x}$

and with Eq. (2) we obtain

$b_{y}=\mu_{0} \frac{\Sigma_{P}}{\Sigma_{H}} J_{H y}$

This provides a direct proportionality between the residual of the $B_{y}$-signal in satellite measurements and the electrojet strength.

A third parameter, that may be used to characterise the electrojet strength, is the merging electric field, $E_{m}$. It is defined as

$E_{m}=v_{S W} \sqrt{B_{y_{\mathrm{IMF}}}^{2}+B_{z_{\mathrm{IMF}}}^{2}} \sin ^{2}\left(\frac{\theta}{2}\right)$

where $v_{S W}$ is the solar wind velocity, $B_{y_{\mathrm{IMF}}}$ and $B_{z_{\mathrm{IMF}}}$ are the interplanetary magnetic field components in GSM coordinates and $\theta$ is the clock angle of the IMF $\left(\theta=\arctan \left(B_{y_{\mathrm{IMF}}} / B_{z_{\mathrm{MF}}}\right)\right)$. The coupling parameter, $E_{m}$, is derived from merging theory assuming that the electric fields in the solar wind mapped down to the day side auroral region are proportional to the ones at the magnetopause (e.g. Kan and Lee, 1979; Boyle et al., 1997). In a statistical study Wang et al. (2005) showed that auroral current intensities on the dayside are well correlated with the strength of $E_{m}$, as long as it stays below the saturation electric field which is of the order of $10 \mathrm{mV} / \mathrm{m}$. For the quiet periods considered here we may write

$J_{H} \sim \Sigma_{H} f_{s} E_{m}$

where $f_{s}$ is a scaling factor representing the mapping geometry from the magnetopause to the ionospheric cusp. According to the T01 field geometry (Tsyganenko, 2002a, b) its value depends on the location of the merging site. It varies between 30 at the subsolar point and 60 for reconnection closer to the flanks. On the dayside the ionospheric conductance is determined predominantly by the solar irradiation. Thus it can be estimated quite reliably in this time sector from the solar zenith angle (e.g. Schlegel, 1988; Moen and Brekke, 1993).

In this chapter we have introduced three different proxies, which can be deduced from observations, independently of the total field (see Eqs. (7), (8), (12), and (14)). They may be suitable for assessing the level of electrojet activity and thus for predicting the modification of the geomagnetic field magnitude by ionospheric currents.

\section{Dataset and processing approach}

For the study of the quiet time current systems we choose 40 days of CHAMP satellite readings centered at winter solstices. The time intervals last from 1 December to 10 January in the northern hemisphere and from 1 June to 10 July in the southern hemisphere. The northern dataset comprises the December solstices 2000-2004, the southern dataset comprises the June solstices 2001-2005, i.e. five seasons in each one.

The ionospheric Hall currents are determined from the scalar magnetic field data of the Overhauser magnetometer on CHAMP. In order to isolate the magnetic effect of ionospheric currents in the CHAMP measurements, the contributions from all other sources are removed from the scalar field readings. Accordingly, the data processing includes subtraction of the main field model CO2 (Holme et al., 2003), employed up to degree and order 14, and elimination of the ring current effect ( $D_{s t}-$ correction), using the external set of coefficients of the same model. We also apply a trend correction along the polar orbit segment to account for possible asymmetries of the ring current, and a parabolic tapering of the currents at the edges of the interval in order to suppress any spurious currents at lower magnetic latitudes $\left(\left|\theta_{m}\right|<50^{\circ}\right)$. The lithospheric magnetisation is accounted for by subtracting a recent crustal magnetic field model, MF3 (Maus et al., 2002). All models used for data correction are consistently based on CHAMP and Ørsted data and are developed from the main field model Oersted-10b-03 (Olsen, 2002). 

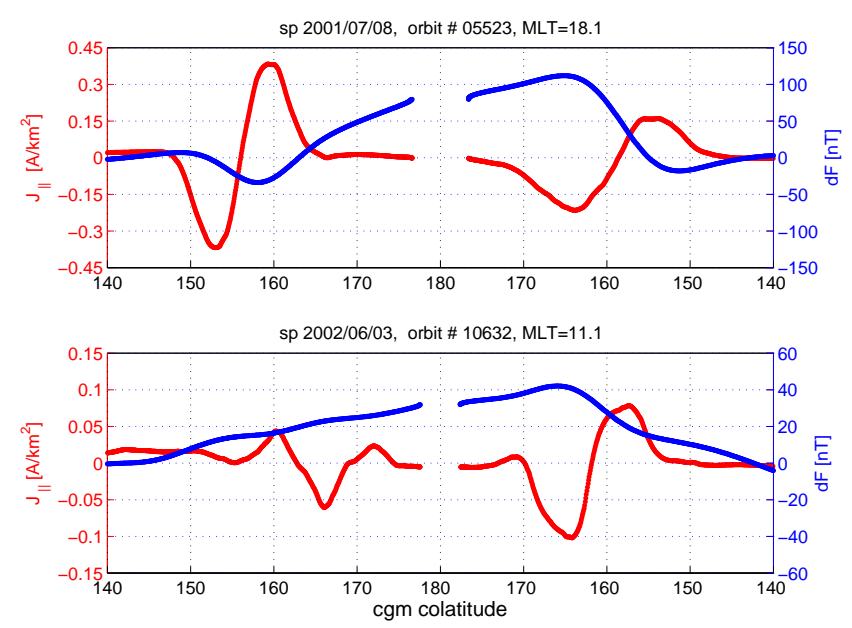

Fig. 2. Field-aligned current, $j_{\|}$(red), and scalar magnetic field deflection, $d F$ (blue), of two example passes across the South Pole. The top track is a dawn-dusk pass with relatively strong amplitudes, the bottom track runs from noon to midnight, in the graph from left to right. It shows weaker signatures. The MLT time refers to the entry of the satellite into the displayed polar region at $140^{\circ} \mathrm{cgm}$ colatitude.

The remaining scalar magnetic field residuals, $d F$, are used for calculating the Hall current density. The horizontal currents are approximated by a series of infinite line currents (Olsen, 1996; Ritter et al., 2004b) and the individual current strengths are found by data inversion of $d F$. The scalar magnetic field residuals are available at a rate of $1 \mathrm{~Hz}$, which is equivalent to 16 measurements per degree of latitude. For the inversion the line currents are placed at a height of $110 \mathrm{~km}$ above the surface of the Earth. They are separated horizontally by $1^{\circ}$ in latitude (also approx. $110 \mathrm{~km}$ ) over an orbit segment of $\pm 80^{\circ}$ centered on the closest approach to the geographic pole. For these calculations a static current system is assumed for the time of passage. Due to the orbital velocity of $4^{\circ}$ in latitude per minute an averaging effect of approximately 5 min occurs (Ritter et al., 2004b).

The FACs are computed from the vector data of the Fluxgate magnetometer. For this procedure the vector components are rotated into the the Mean-Field-Aligned (MFA) coordinate system. The field-aligned currents are computed track-by-track using Ampere's law, $\nabla \times \mathbf{B}=\mu_{\mathbf{0}} \mathbf{j}$. We assume FAC current sheets being aligned with the mean auroral oval (Wang et al., 2005). Then the FAC density, $j_{\|}$, can be estimated as

$j_{\|}=-\frac{1}{\mu_{o} v_{x}} \frac{d B_{y}}{d t}$

Note that for observed field-aligned current densities the symbol $j_{\|}$is used, whereas the model FAC in Sect. 2 are denoted $j_{z} \cdot v_{x}$ is the orbital velocity component perpendicular to the current sheet, accounting for the spatial gradient in that direction. $B_{y}$ is the magnetic field component parallel to the current sheet in the projected MFA x-y plane. According to our assumption the spatial field gradient in $\mathrm{x}$-direction vanishes. Since we cannot compute the true spatial gradients from single-satellite data, this method generally underestimates the current density (Lühr et al., 1996).

Field-aligned currents are known to cover a wide range of spatial scales. In general, higher current densities are encountered for the smaller scale currents (e.g. Ritter and Lühr, 2006). In this study we are aiming at a comparison between the derived FAC density and the residuals of the total field, $d F$ (cf. Eqs. (7) and (8)). Since $d F$ is affected by the electrojet only from a distance of about $300 \mathrm{~km}$ below the satellite, no small-scale features can be resolved. In order to restrict the comparisons between the FAC density and the $d F$ variation to a common wavelength range implied by the distance to the ionospheric Hall current, both series have been highpass filtered with a cut-off period of $100 \mathrm{~s}$. This period converts together with the satellite velocity to a wavelength of about $6^{\circ}$ in latitude.

As another proxy for the electrojet strength we identified the magnetic field component, $b_{y}$, aligned with the FAC sheet (cf. Eq. 12). Here, we apply the same assumption as for the FAC calculation, thus employing directly the transverse component that is aligned with the mean auroral oval.

Our third proxy for estimating the auroral activity is the merging electric field, $E_{m}$. It is derived exclusively from solar wind parameters, namely the IMF components and the solar wind velocity. These data with $1 \mathrm{~min}$ resolution are obtained from the Advanced Composition Explorer (ACE) satellite (final calibration). The transit time of each event to the sub-solar magnetopause is calculated from the measured solar wind speed. For the development of the FAC and the propagation to the ionosphere we add another $15 \mathrm{~min}$ (e.g. Vennerstrøm et al., 2002). Despite these corrections a significant uncertainty in the arrival time of solar wind features is left.

\section{Statistical properties of the quiet time currents}

It has been shown by Ritter et al. (2004a) that the majority of quiet polar passes are confined to the season when the pole is continuously in darkness. Here, we make use of this fact and limit our investigations to the 40 days centred around the respective winter solstice. Considering the orbital period of CHAMP $(\approx 93 \mathrm{~min})$ this time interval provides about 620 passes per season. If we multiply that by the five available years the resulting number of tracks is sizeable and forms a good basis for a statistical study.

\subsection{Relation between FAC and $d F$}

As has been shown in Sect. 2, a close relation is expected between the variations of the FAC density and the residuals of the magnetic field magnitude. Figure 2 shows two example 

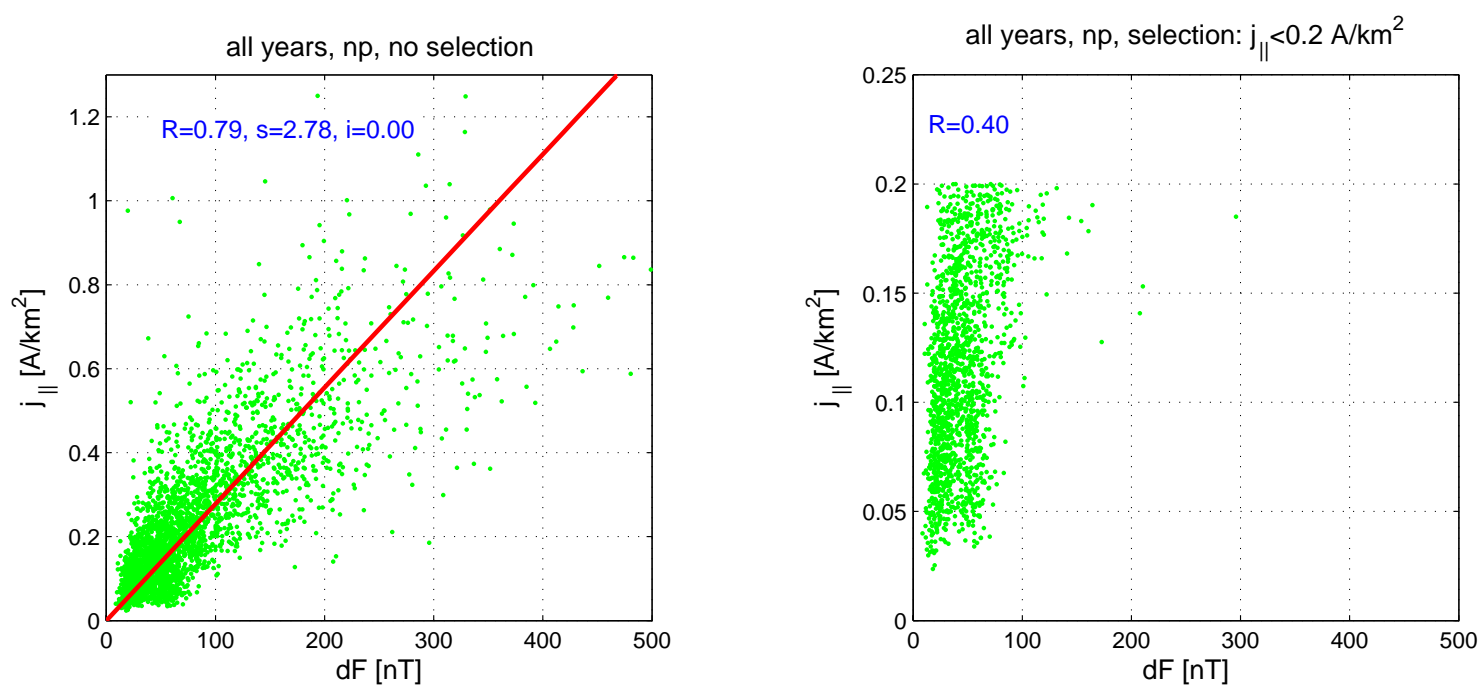

(a)

(b)

Fig. 3. Correlation of the peak-to-peak values of $j_{\|}$and $d F$ of all dark season passes at the North Pole (left). The right side diagram shows only small events with $j_{\|}<0.2 \mathrm{~A} / \mathrm{km}^{2}$. $R$ : correlation coefficient, $s, i$ : slope and intercept of the regression line (bivariate).

passes over the South Pole. In the top panel CHAMP passes from dusk to dawn (from left to right). The red curve depicts the FAC density. Positive values represent upward currents. Here, the classical Region1/Region 2 configuration can be recognized quite clearly. The blue curve, representing the deviation of the field magnitude, exhibits the typical bi-polar variation: negative deflections at dusk, positive at dawn. In general, there is a tendency of an anti-correlation between the variations of the two curves, as expected from Eq. (3). However, the scaling factors between the two quantities are different in the two time sectors. The lower panel shows the situation for a pass from noon to midnight. Here, things are less clear. The electrojet activity around noon seems to be strongly suppressed, whereas sizeable currents are flowing on the night side. As a consequence, the typical $d F$ signature is degraded. Only a positive deflection peaking on the night side is left. Nevertheless, it influences the total field all the way into the noon sector of the auroral zone.

In order to get a more quantitative impression of the relation between the FAC density and the $d F$ deflections, a correlation analysis was performed. Figure $3 \mathrm{a}$ shows the relation of the peak-to-peak values of these two quantities in a scatter plot for all available North Pole passes (winter season). The good correlation between the FAC density and the variation of the total field is supported by the correlation coefficient, $R=0.79$. From the slope of the regression line, $s$, we can deduce an average relation for the dark ionosphere

$j_{\|_{\max }}=2.8 \cdot d F_{\max }$
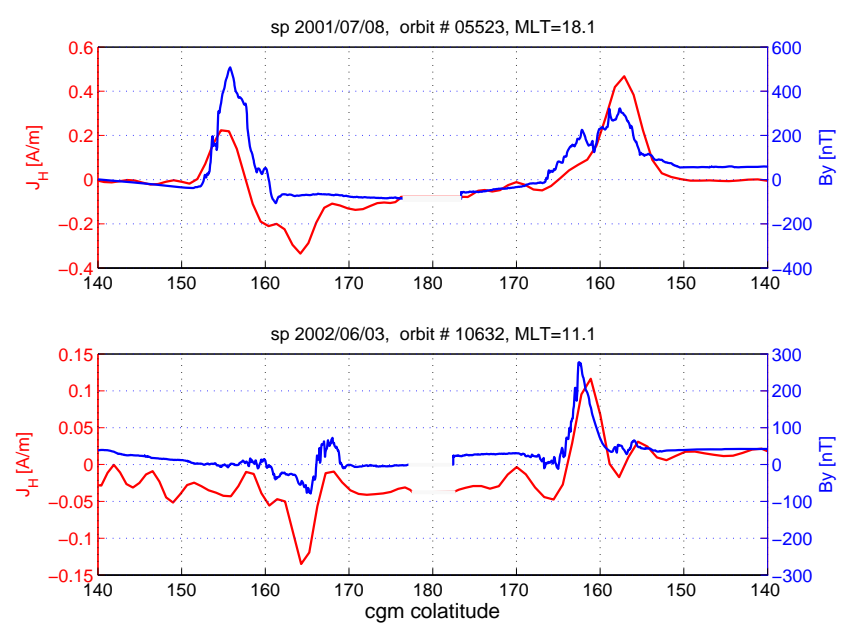

Fig. 4. Transverse magnetic component, $B_{y}$ (blue), and Hall current, $J_{H}$ (red), of the same two example passes as in Fig. 2.

where $j_{\|}$is measured in $\mathrm{mA} / \mathrm{km}^{2}$ and $d F$ in $\mathrm{nT}$. When combining Eqs. (7) and (8) we get

$j_{\|_{\max }}=\frac{\Sigma_{P}}{\Sigma_{H}} \frac{2}{\mu_{0}} \frac{(h+a)}{a^{2}} d F_{\max }$

If we consider the factor 2.8 of Eq. (16), take a height of $h=300 \mathrm{~km}$ above the current sheet and a ratio $\Sigma_{H} / \Sigma_{P}=2$ (Davies and Lester, 1999) we obtain for the half peak width of the electrojet $2 a \approx 930 \mathrm{~km}$. This is a reasonable number for the latitudinal width of the current distribution, as seen from 


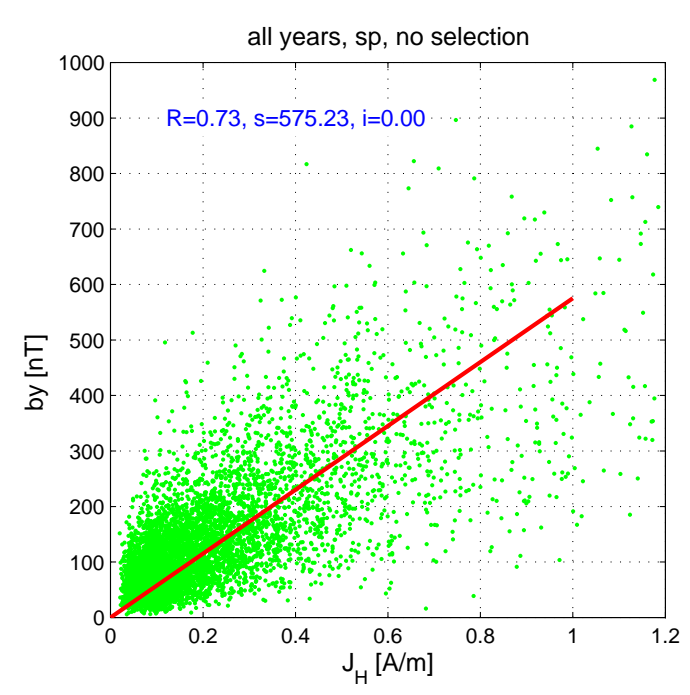

(a)

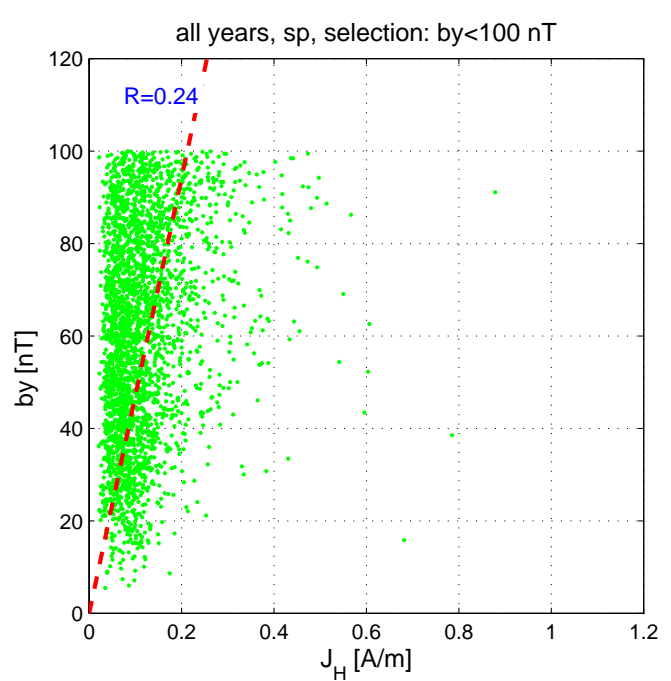

(b)

Fig. 5. Correlation of the peak-to-peak values of $B_{y}$ and $J_{H}$ of all dark season passes at the South Pole (left). The right side diagram shows only small events with $b_{y}<100 \mathrm{nT}$. $R$ : correlation coefficient, $s, i$ : slope and intercept of the regression line (bivariate).

CHAMP. From these results we may conclude that, in general, the FAC density is a suitable indicator for the expected deviations of the field magnitude.

The prime purpose of this study, however, is to identify passes with small magnetic perturbation. The mean FAC density in Fig. 3a is $0.24 \mathrm{~A} / \mathrm{km}^{2}$, and approximately $60 \%$ of the data have amplitudes below this value. So we try to use a maximum FAC density of $j_{\|}<0.2 \mathrm{~A} / \mathrm{km}^{2}$ as a possible selection criterion. As can be seen from the scatter plot, below this threshold $\mathrm{dF}$ deflections are quite small, mostly below $100 \mathrm{nT}$, or even below their mean amplitude of $60 \mathrm{nT}$. The distribution obtained for this limited range is shown in Fig. 3b. Unfortunately, the good correlation does no longer exist. Even for these weak field-aligned currents variations in the field strength well beyond $100 \mathrm{nT}$ can be observed. Such disturbances may vitiate modelling studies.

\subsection{Relation between Hall current density and $B_{y}$-deflection}

According to Eq. (12) there should exist a close relationship between the Hall current intensity and the magnetic deflection of the component parallel to the current sheet. Typical examples of such a comparison are shown in Figs. 4. Again, the upper panel presents a pass from dusk to dawn and the lower one a pass from noon to midnight. As expected, the variations track each other quite closely, although there are some differences in the details. This is no surprise, as $B_{y}$ is a rather local measurement while the estimate of the Hall current flowing some $300 \mathrm{~km}$ below is an average value over a larger area. The resolution of our Hall current estimates was found to be $0.04 \mathrm{~A} / \mathrm{m}$ (Ritter et al., 2004b).
When comparing the amplitudes of the blue and red curves, there seem to be differences in the four time sectors covered by the two examples (dawn, dusk, noon, midnight). Therefore, we looked closer into the ratios between $B_{y}$ and $J_{H}$. Figure 5a shows a scatter plot of the peak-to-peak values of the transverse field deflection versus the Hall current density for all South Pole passes (winter season). Again, a reasonably good correlation with $R=0.73$ emerges. According to Eq. (12) the slope of the regression line can be used to estimate the average ratio between the conductances, $\Sigma_{P} / \Sigma_{H}$. From the regression line we obtain

$\mu_{0} \frac{\Sigma_{P}}{\Sigma_{H}}=575 \cdot 10^{-9}$

For the conductance ratio this analysis yields $\Sigma_{H} / \Sigma_{P}=2.2$, which is a reasonable value. Here again, it is tested how well the transverse magnetic deflection, $B_{y}$, can be used to identify passes with weak electrojets. The mean amplitude of all peak-to-peak magnetic deflections, denoted $b_{y}$, is $165 \mathrm{nT}$. Again, as a threshold we used a value below this mean value, $b_{y}<100 \mathrm{nT}$. This threshold confines the majority of the Hall current intensities below their mean amplitude of $0.3 \mathrm{~A} / \mathrm{m}$. Figure $5 \mathrm{~b}$ shows the distribution of this truncated distribution. There is hardly any correlation left when the transverse field deflections are limited to small amplitudes. A sizable amount of cases exhibit current densities beyond $0.4 \mathrm{~A} / \mathrm{m}$. According to Eq. (8) this converts to an approximate variation of the field strength, $d F \approx 100 \mathrm{nT}$ peak-to-peak. At this point it turns out that the transverse field amplitude is also not a reliable indicator for low electrojet activity. 

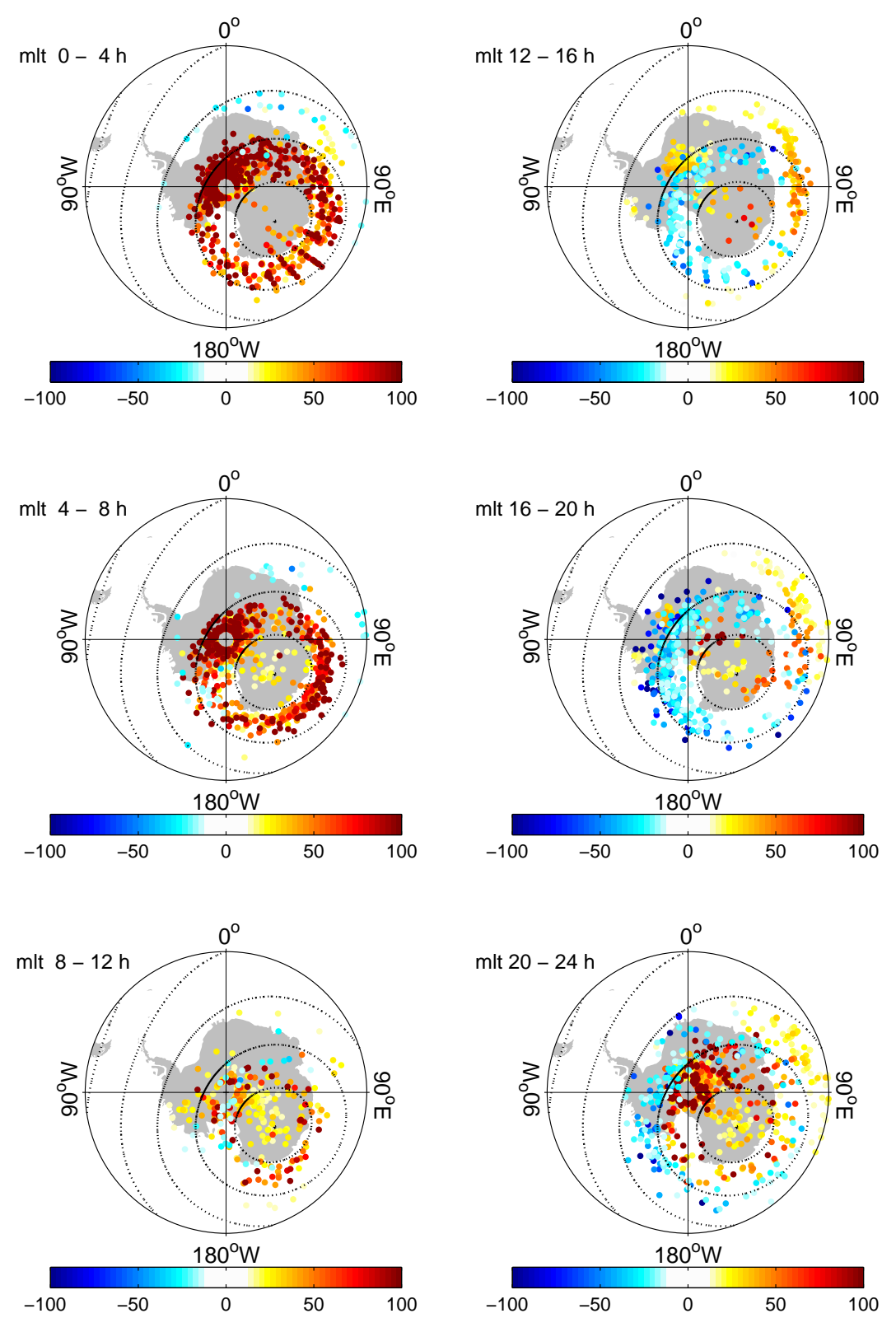

Fig. 6. Minima and Maxima of all magnetic deflections, $d F$, sorted by magnetic local time (MLT), South Pole. The colours represent [nT]. Dashed circles mark cgm latitudes in steps of $10^{\circ}$. Only $|d F|>10 \mathrm{nT}$ are plotted.

\subsection{Local time dependence of $d F$}

It is well-known that auroral currents show a distinct local time pattern. This reflects to a large extent the processes taking place at conjugate locations in the outer magnetosphere. For that reason we may expect that the relation between our selected activity proxies and the deflections of the total field, $d F$, shows also different values in the various time sectors. This assumption is supported by observations of Wang et al. (2005) who found a strong seasonal dependence of the auroral currents on the dayside but little dependence on the night side.
To illustrate the MLT dependence during the dark season Fig. 6 shows the distribution of $d F$ peak values for six different time zones at the South Pole. From the colour-coding of the amplitudes the typical distribution of auroral electrojets becomes evident. During the hours after midnight the red dots dominate all along auroral latitudes. These positive deflections are indicative for a westward electrojet. During the four hours before noon, there is a mixture of positive and negative deflections. In this sector, the direction of the electrojet is quite variable. In addition, the number of dots is smaller. This is an indication of weak currents as only 

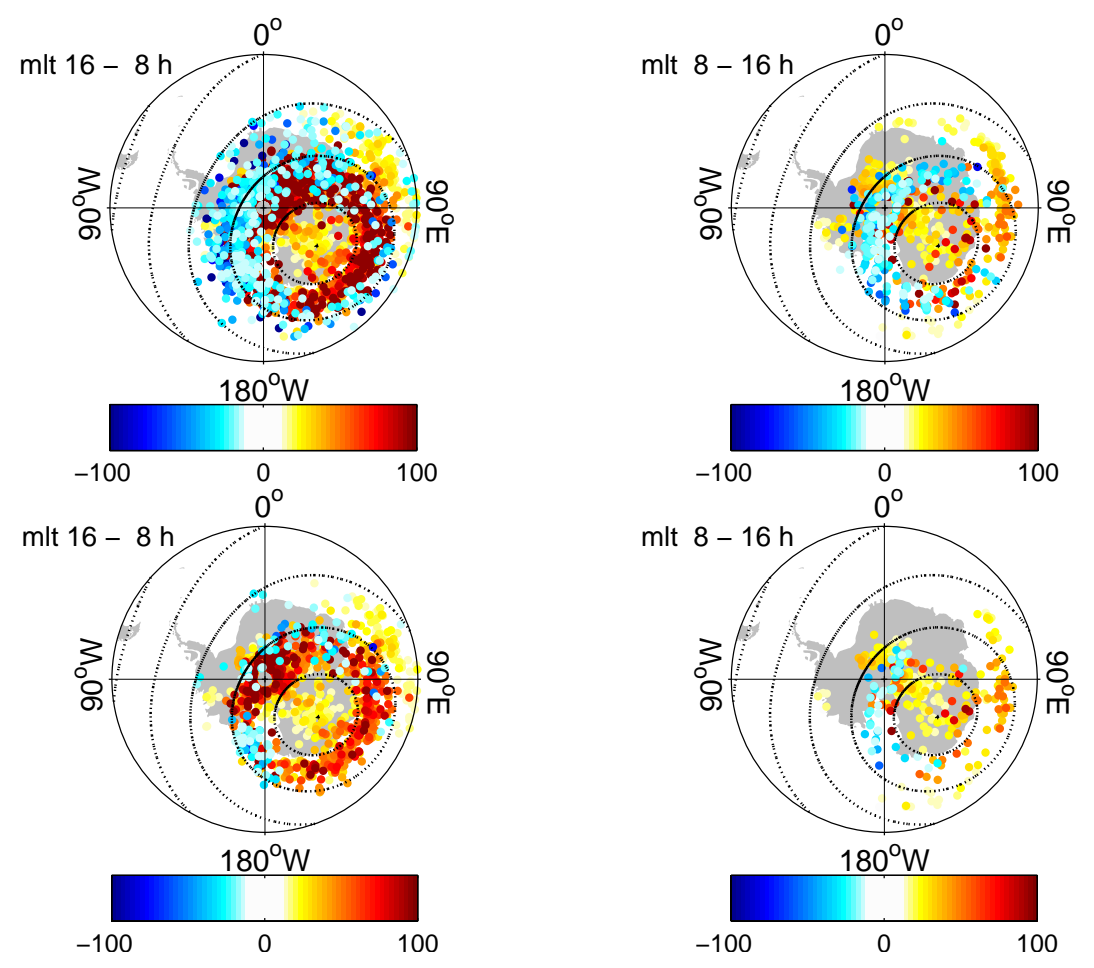

Fig. 7. Minima and Maxima of $d F$ [nT], sorted by MLT into nightside (16:00-08:00 h, left) and dayside (08:00-16:00 h, right), South Pole. Top frames: all events; bottom frames: only events with $j_{\|}<0.2 \mathrm{~A} / \mathrm{km}^{2}$.

$|d F|>10 \mathrm{nT}$ are plotted. After noon, the blue dots dominate showing the dominance of the eastward electrojets. During the hours before midnight, we find a co-existence of the eastward and westward electrojets, the latter is found more poleward.

After having seen the distribution of $d F$ for all passes we applied selection criteria for finding the passes with minimum deflections. As a first criterion we limit the time sector to dayside hours, i.e. 08:00-16:00 MLT. During these hours, we find the smallest amplitudes in Fig. 6. The top row of Fig. 7 shows the peak values sorted into two MLT bins only: 08:00-16:00 $\mathrm{h}$ and 16:00-08:00 $\mathrm{h}$. As an additional condition we request the FAC density to be less than $0.2 \mathrm{~A} / \mathrm{km}^{2}$. The bottom row of Fig. 7 shows the application of this threshold for the two MLT ranges. The number of available passes in the dayside bin is not only largely reduced compared to the previous MLT sorting, but also the amplitude of $d F$ is much smaller within the selected subset. The rejected time sector (nightside) contains clearly larger deflections.

We also tested the second criterion, i.e. the peak deflection of the transverse component, $B_{y}$. The threshold applied is by $<100 \mathrm{nT}$. Combining this criterion with the dayside hours 08:00-16:00 MLT provides a distribution of $d F$ amplitudes, as shown in Fig. 8. Now, the number of passes in our selected time window is larger, and the events with $d F>50 \mathrm{nT}$ are even more frequent. Apparently, our $B_{y}$-criterion seems to be less efficient in selecting the undisturbed passes. We varied the threshold of $\Delta B y$, but the ratio between selected passes and those with $d F>50 \mathrm{nT}$ stays worse than the yield by the FAC criterion.

The results of these tests are not regarded as fully satisfying, presumably because of the simplifying assumptions built into the models of electrojets and FACs. Even though the selection by FAC density yields better results, there remains a general uncertainty inherent to FAC estimates based on single spacecraft and the assumption of uniform conductivity. In the selection process a number of passes could not be considered at all, as their angle between current sheet and satellite orbit falls below $30^{\circ}$. At such small angles the FAC estimates become very unreliable.

\subsection{Relation between the merging E-field and $d F$}

In their earlier study Ritter et al. (2004a) demonstrated that there is a close relationship between quiet polar passes and the strength of the solar wind merging electric field, $E_{m}$. After having tried the local activity indicators we are interested how efficiently this remote parameter can be used to find quiet passes. In contrast to the above mentioned publication we limit our investigations here to the dayside hours 08:00-16:00 MLT. These were shown to respond most directly to $E_{m}$ by Wang et al. (2005). 

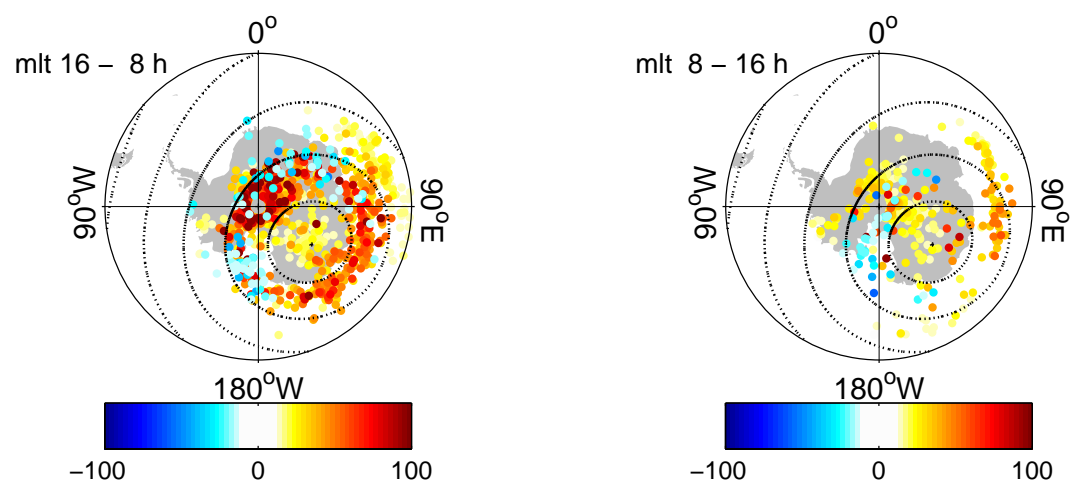

Fig. 8. Minima and Maxima of $d F$ [nT], left frame: nightside (16:00-08:00 h); right frame: dayside (08:00-16:00h), only events with by $<100 \mathrm{nT}$, South Pole.

In Sect. 2 we suggested a linear relation between the merging electric field and the Hall current density (cf. Eq. 14). In order to test this assumption the relation between the two quantities is plotted. Figure 9 shows the results of a correlation analysis for passes within the time sector 08:0016:00 MLT. The scatter is quite large and therefore the result is not too significant. Nevertheless, we may use the slope $s$ of the regression line to estimate the relation between $E_{m}$ and $J_{H}$.

$E_{m} \approx 5.9 \cdot 10^{-3} J_{H}$

When comparing this number with Eq. (14) and using an average $f_{S}$ of 45 we obtain a Hall conductance of $\Sigma_{H} S=3.7$, which is a reasonable value for the dark hemisphere.

Having verified a relationship between $E_{m}$ and the auroral current strength we tested several criteria for identifying quiet periods. The first one follows the suggestion of Ritter et al. (2004a) to use a limit of $E_{m}<0.8 \mathrm{mV} / \mathrm{m}$ as an average value over an interval of $40 \mathrm{~min}$ centered around the pole passage. The distribution and amplitude of the peak deflections in $d F$ are shown in Fig. 10. In the selected dayside hours (right panel) the amplitudes are much smaller than during the night.

Some of the passes in the right frame still exhibit amplitudes beyond $50 \mathrm{nT}$. We suggest that the variability of $E_{m}$ during the $40 \mathrm{~min}$ interval considered could be quite large and cause temporary current enhancements. In order to exclude those passes we added the requirement that the peak value of $E_{m}$ should not exceed $1.2 \mathrm{mV} / \mathrm{m}$ during the $40 \mathrm{~min}$ interval. This threshold is also taken from the statistical study of Ritter et al. (2004a) and marks the $E_{m}$ amplitude beyond which the occurrence rate of $E_{m}$ values of quiet passes drops below $50 \%$ compared to that of the complete dataset. This requirement removes many of the outliers (see Fig. 11, top part). The remaining distribution of $d F$ peaks seems to be unrelated to auroral processes. They reflect probably more crust-related anomalies which have not been accounted for by the applied model. On the night side, there are still size-

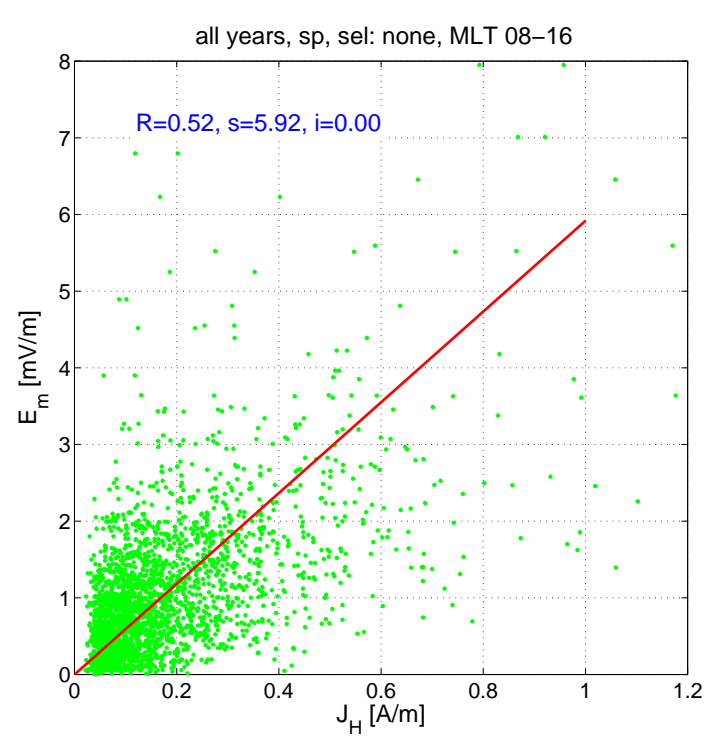

Fig. 9. Correlation plot of the average merging electric field, $\bar{E}_{m}$, and the peak-to-peak values of the Hall currents, $J_{H}$, of the dayside passes (08:00-16:00 MLT), South Pole. $R$ : correlation coefficient, $s, i$ : slope and intercept of the regression line (bivariate).

able, predominantly positive deflections to be observed. This means westward electrojets are flowing even during times of low solar wind input.

So far, we presented the local time dependence of the auroral activity for the southern hemisphere. In the northern hemisphere, quite similar results were obtained. The lower part of Fig. 11 shows the distribution of deflections in the total field for the combined selection criteria of $E_{m}$, average value below $0.8 \mathrm{mV} / \mathrm{m}$ and peak value below $1.2 \mathrm{mV} / \mathrm{m}$, in the northern polar region. Again, the dayside sector is free of aurora-related disturbances while on the nightside the oval is well marked by peak deflections. Interestingly, there is a distinct patch of positive deflections north of Canada, 

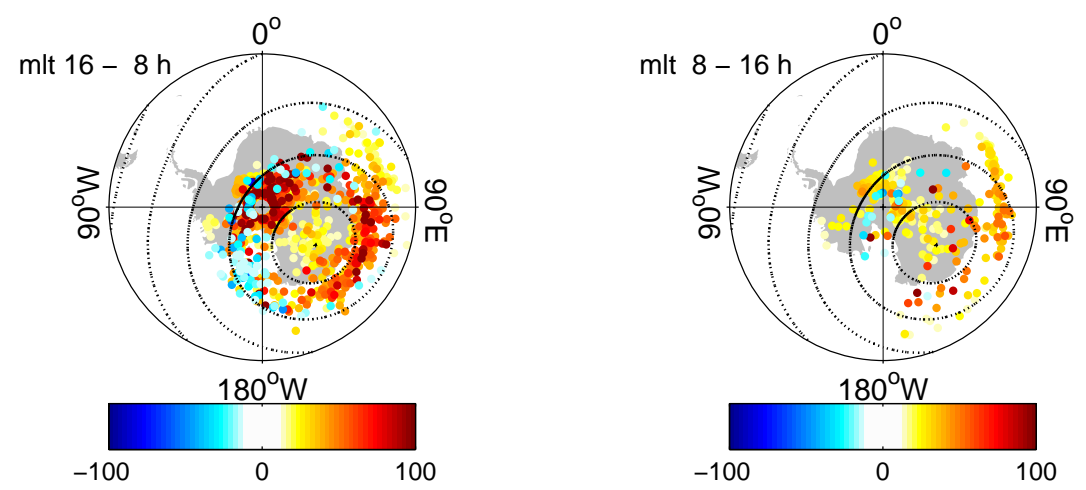

Fig. 10. Minima and Maxima of $d F$ [nT], left frame: nightside (16:00-08:00 h); right frame: dayside (08:00-16:00 h), only events with average $\bar{E}_{m}<0.8 \mathrm{mV} / \mathrm{m}$, South Pole.
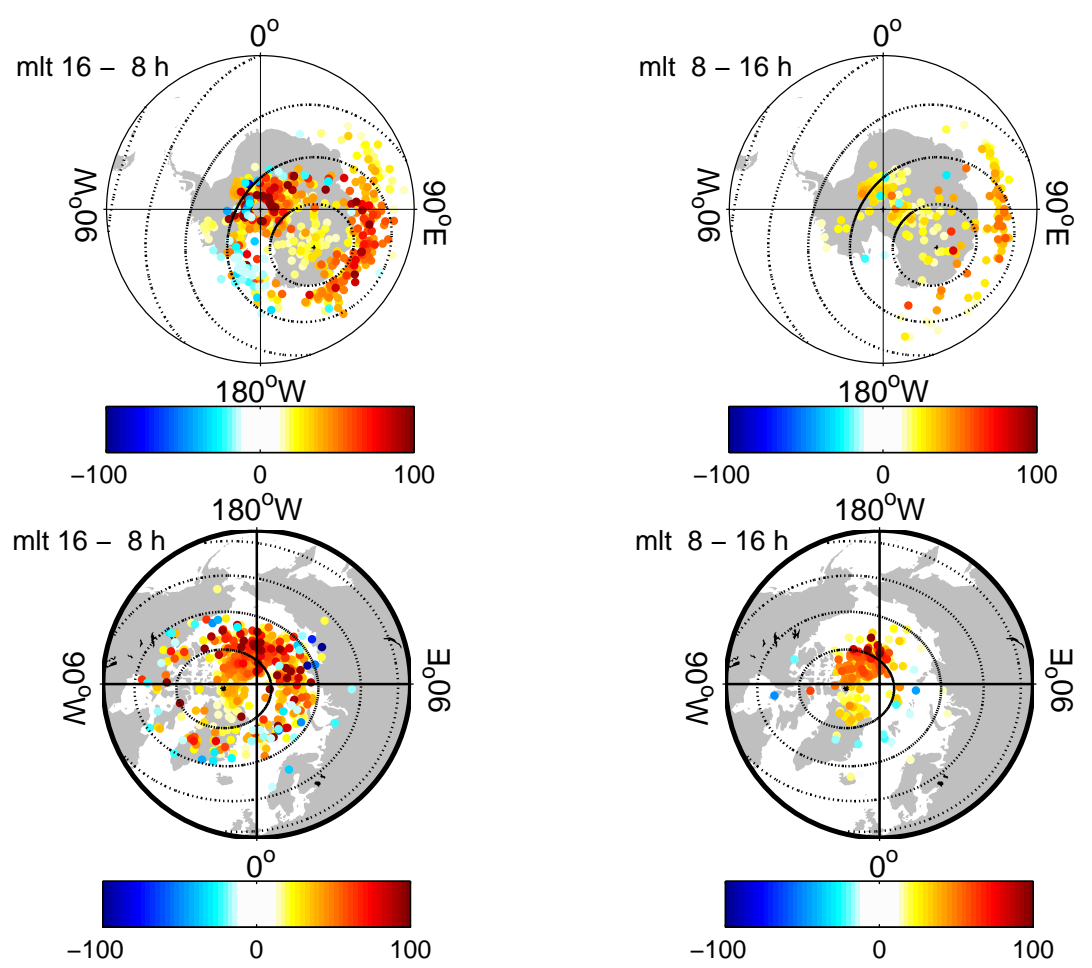

Fig. 11. Minima and Maxima of $d F[\mathrm{nT}]$, left frames: nightside (16:00-08:00 h); right frames: dayside (08:00-16:00h), only events with average $\bar{E}_{m}<0.8 \mathrm{mV} / \mathrm{m}$ and peak $\hat{E}_{m}<1.2 \mathrm{mV} / \mathrm{m}$ during the hemisphere crossing (40 min). Top: South Pole; Bottom: North Pole.

centered at approximately $135^{\circ} \mathrm{W}, 80^{\circ} \mathrm{N}$. There seems to be a prominent magnetic anomaly which is not sufficiently accounted for by the magnetic field model used in this study. A positive magnetic anomaly can indeed be observed in recent crustal anomaly maps e.g. by Maus et al. (2006) and Thebault (2006).

The finally evolved selection criteria that impose limits on the merging electric field, the season and the local time have been shown to work well in both hemispheres.

\section{Discussion}

The prime purpose of this study is the investigation of auroral electrodynamics during quiet periods. It is not a simple task to identify low activity intervals from common indices such as $K_{p}, D_{s t}$ or $A E$. We tried a different approach, using locally determined current parameters. For the modelling of the internal magnetic field it is most important to use magnetic field data from satellite passes that are least disturbed by ionospheric currents. An important modulator of the auroral 
Table 1. Quiet period detection yield for the various selection criteria presented in Sects. 4.1-4.4.

\begin{tabular}{|c|c|c|c|c|}
\hline \multirow[b]{2}{*}{ selection criterion } & \multicolumn{2}{|r|}{ South Pole } & \multicolumn{2}{|c|}{ North Pole } \\
\hline & all MLT & 08:00-16:00 MLT & all MLT & 08:00-16:00 MLT \\
\hline none & 5417 & 1465 & 5369 & 1529 \\
\hline$j_{\|}<0.2 \mathrm{~A} / \mathrm{km}^{2}$ & 2811 & 716 & 2969 & 1073 \\
\hline$b_{y}<100 \mathrm{nT}$ & 2486 & 767 & 2099 & 783 \\
\hline$\vec{E}_{m}<0.8 \mathrm{mV} / \mathrm{m}$ & 2410 & 679 & 2082 & 679 \\
\hline $\bar{E}_{m}<0.8 \mathrm{mV} / \mathrm{m} \& \hat{E}_{m}<1.2 \mathrm{mV} / \mathrm{m}$ & 1770 & 502 & 1462 & 503 \\
\hline
\end{tabular}

activity is the amount of solar illumination of the polar region. Therefore, we limited our study to 40 days centered around the hemispheric winter solstices. It is common practise that at high latitudes only the field magnitude is used for main field modelling. The idea was therefore to use the signature of the transverse components that are not considered in the modelling for predicting the total field variations. Another underlying assumption is that, at high latitudes, shorter scale crustal anomalies cause only relatively small deflections in the transverse components. Therefore, no generic crustal signal would be removed by this procedure.

In a first test we used the FAC density as an indicator for the auroral activity. As is shown in Sect. 2, a close relation between the FAC density and the deviation of the total field is expected. When considering data from all days the correlation analysis confirms the expected relation between these two quantities. Based on this dependence it was hoped that FAC estimates can be used to select passes with low deflections of the total field. However, when limiting the dataset to cases with small FAC densities, $j_{\|}<0.2 \mathrm{~A} / \mathrm{km}^{2}$, the correlation with total field variations breaks down. Our explanation for this drop of correlation is that the intensity of the electrojet causing the $d F$ deflection is not only dependent on local FAC strength but is also influenced by FAC closing further away. These signatures, however, are not detected by the single satellite FAC estimation method used here. Another limitation of this method is that FAC densities cannot be determined uniquely. Assumptions have to be made on the geometry of the FAC sheets. Uncertainties generally result in underestimations of the FAC density (Lühr et al., 1996). For all these reasons the local FAC density can in general not be considered a suitable indicator for quiet polar passes.

As another proxy for the auroral current activity we used the deflection of the transverse magnetic component, aligned with the auroral oval. This field component, $b_{y}$, has been shown to be, under certain conditions, proportional to the Hall currents of the electrojet. Here again, the correlation analysis confirms a linear relationship when data of all days are considered. The slope of the regression line can be used to derive the average ratio between the Hall and Pedersen conductances. The estimated value, $\Sigma_{H} / \Sigma_{P}=2.2$, is slightly higher than the average as estimated by Davies and Lester (1999). Here, we have to keep in mind, that the slope of the regression line fitted to the data scatter is determined mainly by the strong events. It is known that the ratio between the conductances is elevated during disturbed periods (e.g. Schlegel, 1988). When limiting the attention to weaker events, the degree of correlation is severely reduced preventing a reliable calculation of the regression line. We therefore cannot check whether the conductance ratio is close to 1 , as one would expect.

To achieve our prime goal, the selection of quiet-time polar passes, we considered only cases with small peak-to-peak deflections, $b_{y}<100 \mathrm{nT}$. The explanation for the poor correlation is the same as before. The deflection, $b_{y}$, is a local measurement, but the Hall current is influenced by inputs over a larger area. Therefore, also this indicator cannot be used straight away for identifying periods of low activity.

In our quest to single out undisturbed satellite passes we investigated the local time dependence of the activity. Wang et al. (2005) had found already, that the seasonal dependence of the auroral current strength is virtually confined to the dayside sector. In agreement with this conclusion we find the smallest deflections in the total field during the pre-noon hours. Based on these observations we tested the efficiency of the indicators introduced above, when the data sampling is limited to the dayside sector, 08:00-16:00 MLT. The result is quite promising for the FAC criterion, $j_{\|}<0.2 \mathrm{~A} / \mathrm{km}^{2}$. There are only rather few passes exhibiting deflections with amplitudes exceeding $50 \mathrm{nT}$. Our transverse field criterion, $b_{y}<100 \mathrm{nT}$, is less effective in detecting the quiet passes.

Another quantity reflecting the solar wind input to the magnetosphere is the merging electric field, $E_{m}$. It was already identified by Ritter et al. (2004a) as a useful indicator for auroral activity. When applying the limitation, $E_{m}<0.8 \mathrm{mV} / \mathrm{m}$ to the satellite passes, as suggested there, within the time sector 08:00-16:00 MLT, a rather good selection of quiet passes results. Since the calculation of $E_{m}$ is based on ACE data propagated to the magnetopause, there is an uncertainty in the timing of the solar wind data with respect to ionospheric observations. To account for this uncertainty, we used averages of $40 \mathrm{~min}$ centered at the time 
of the ionospheric observation. In a further step we require that $E_{m}$ should always be below $1.2 \mathrm{mV} / \mathrm{m}$ during the $40 \mathrm{~min}$ time interval. The additional truncation reduced the number of passes with excessive magnetic deflection efficiently.

At this point we investigate which fraction of the available CHAMP passes are identified as quiet. Table 1 lists the occurrence numbers for the various criteria individually for the two poles. In total about 5500 orbit arcs are available in each hemisphere. When applying the small-FAC criterion combined with the limited MLT sector, approximately 13-20\% are passing as quiet. The yield is significantly smaller at the South Pole than at the North Pole. This is to a good part be due to the fact that in the southern hemisphere, many more passes with shallow angles to the auroral oval $\left(<30^{\circ}\right)$ occur. These are rejected from FAC calculation. The $b_{y}$ criterion shows a yield almost equally distributed in local time and amongst the poles. The selection rate of this method is in the range of the FAC criterion rate (15\%).

When using the merging electric field for sorting the data, the yield is slightly reduced. The threshold value $\bar{E}_{m}<0.8 \mathrm{mV} / \mathrm{m}$, as proposed earlier, selects $13 \%$ of the passes, equally in both hemispheres. With the refinement of an additional truncation of orbit arcs with peak values $\hat{E}_{m}>1.2 \mathrm{mV} / \mathrm{m}$, we singled out about 500 passes for each polar region. This is $10 \%$ of the dark season data studied here, but slightly less than $2 \%$ of the total number of passes in the investigated time interval of 5 years. Having only 500 passes in 5 years is probably not enough for determining the secular variation of the geomagnetic field, in particular, because our dataset is unevenly distributed in time, alternating between the poles. In case of the lithospheric field, time variations are not expected on decadal scales. For these models the quiet data of all years can be accumulated. We expect this clean dataset can be used beneficially for high degree spherical harmonic expansions.

When looking at the finally selected passes in Figs. 11, right graphs, we notice that, in some areas, the peak values of $d F$ mark persistent features. In magnetic coordinates, these features are coherent. The quiet dataset promises to reveal new crustal features which were not identified in previous models. The positive anomaly north of Canada with amplitudes up to $110 \mathrm{nT}$ is particularly outstanding. The average intensity of this prominent area is $45 \mathrm{nT}$ and clearly exceeds the crustal anomaly computed in this region. Here, the lithospheric models need significant revision. The virtue of the selection criterion proposed in this paper is that it is controlled completely by external parameters i.e. IMF components, MLT and solar zenith angle. There is no risk of a feed-back from previous models on the selection.

A list of the selected quiet CHAMP passes is publicly available at http://www.gfz-potsdam.de/pb2/pb23/Mag/ quiet_polar_passes.html.
Acknowledgements. We are obliged to F. Christiansen of DNSC who kindly provided us with the solar wind data (ACE) propagated to the magnetopause. Furthermore, we thank the ACE MAG and SWEPAM instrument teams and the ACE Science Center for providing the ACE data. The operational support of the CHAMP mission by the German Aerospace Center (DLR) and the financial support for the data processing by the Federal Ministry of Education (BMBF), as part of the Geotechnology Programme, are gratefully acknowledged. The DFG supported PR through the Priority Programme "Geomagnetic Variations" SPP 1097.

Topical Editor M. Pinnock thanks A. Thomson and another referee for their help in evaluating this paper.

\section{References}

Boyle, C. B., Reiff, P. H., and Hairston, M. R.: Empirical polar cap potentials, J. Geophys. Res., 102, 111-125, 1997.

Holme, R., Olsen, N., Rother, M., and Lühr, H.: CO2 - A CHAMP magnetic field model, in: First CHAMP Mission Results for Gravity, Magnetic and Atmospheric Studies, edited by: Reigber, C., Lühr, H. , and Schwintzer, P., Springer, Berlin, 220-225, 2003.

J. A. Davies and Lester, M.: The relationship between electric fields, conductances and currents in the high-latitude ionosphere: a statistical study using EISCAT data, Ann. Geophys., 17, 43-52, 1999 ,

http://www.ann-geophys.net/17/43/1999/.

Kan, J. R. and Lee, L.: Energy coupling function and solar windmagnetosphere dynamo, Geophys. Res. Lett., 6, 577, 1979.

Kertz, W.: Modelle für erdmagnetisch induzierte elektrische Ströme im Untergrund, Nachr. Akad. Wiss. Göttingen Math.-phys. Kl., 5, 101-110, 1954.

Langel, R. and Estes, R.: The near earth magnetic field at 1980 determined from Magsat data, J. Geophys. Res., 90, 2495-2510, 1985.

Lühr, H., Warnecke, J., and Rother, M.: An algorithm for estimating field-aligned currents from single spacecraft magnetic field measurements: a diagnostic tool applied to Freja satellite data, IEEE Trans Geosci. Remote Sens., 34, 1369-1376, 1996.

Maurer, H. and Theile, B.: Parameters of the aurora1 electrojet from magnetic variations along a meridian, J. Geophys., 44, 415-426, 1978.

Maus, S., Rother, M., Holme, R., Lühr, H., Olsen, N., and Haak, V.: First scalar magnetic anomaly map from CHAMP satellite data indicates weak lithospheric field, Geophys. Res. Lett., 29, doi:10.1029/2001GL013685, 2002.

Maus, S., Rother, M., Hemant, K., Stolle, C., Lühr, H., Kuvshinov, A., and Olsen, N.: Earth's lithospheric magnetic field determined to spherical harmonic degree 90 from CHAMP satellite measurements, Geophys. J. Int., doi:10.1111/j.1365246X.2005.02 833.x, 2006.

Moen, J. and Brekke, A.: The solar flux influence on quiet time conductances in the auroral ionosphere, Geophys. Res. Lett., 20(10), 971-974, 1993.

Olsen, N.: A new tool for determining of ionospheric currents from satellite data, Geophys. Res. Lett., 23, 3635-3638, 1996.

Olsen, N.: A model of the geomagnetic main field and its secular variation for epoch 2000 estimated from Ørsted data, Geophys. J. Int., 149, 454-462, 2002. 
Ritter, P. and Lühr, H.: Curl-B technique applied to Swarm constellation for determing field-aligned currents, Earth, Planets and Space, 58(4), 463-476, 2006.

Ritter, P., Lühr, H., Maus, S., and Viljanen, A.: High latiutude ionospheric currents during very quiet times: their characteristics and predictabilities, Ann. Geophys., 22, 2001-2014, 2004a.

Ritter, P., Lühr, H., Viljanen, A., Amm, O., Pulkkinen, A., and Sillanpää, I.: Ionospheric currents estimated simultaneously from CHAMP satellite and IMAGE ground-based magnetic field measurements: a statistical study at auroral latitudes, Ann. Geophys., 22, 417-430, 2004b.

Schlegel, K.: Auroral zone E-region conductivities during solar minimum derived from EISCAT data, Ann Geophys., 6, 129138, 1988.

Thébault, E.: Global lithospheric magnetic field modelling by successive regional analysis, Earth Planets Space, 58, 485-495, 2006.
Tsyganenko, N. A.: A model of the near magnetosphere with a dawn-dusk asymmetry, 1. Mathematical structure, J. Geophys. Res., 107(A8), 1179, doi:10/1029/2001JA000219, 2002a.

Tsyganenko, N. A.: A model of the near magnetosphere with a dawn-dusk asymmetry, 2. Parametrization and fitting to observations, J. Geophys. Res., 107(A8), 1176, doi:10.1029/2001JA000220, 2002b.

Vennerstrøm, S., Moretto, T., Olsen, N., Friis-Christensen, E., Stampe, A., and Watermann, J.: Field-aligned currents in the dayside cusp and polar cap region during northward IMF, J. Geophys. Res., 107(A8), doi:10.1029/2001JA009162, 2002.

Wang, H., Lühr, H., and Ma, S.-Y.: Solar zenith angle and merging electric field control of field-aligned currents: A statistical study of the southern hemisphere, J. Geophys. Res., 110, A03306, doi:10.10292004JA010530, 2005. 